\section{Monthly OCT monitoring of Ozurdex for macular oedema related to retinal vascular diseases: re-treatment strategy (OCTOME Report 1)}

\author{
Abstract \\ Purpose To evaluate the morphological and \\ functional changes following intravitreal \\ Ozurdex (dexamethasone implant) injections \\ in patients with macular oedema (MO) \\ secondary to retinal vascular diseases. \\ Design This is a single centre, exploratory \\ phase III, prospective, open-label clinical \\ study. \\ Methods Thirty patients with MO \\ secondary to retinal vascular disorders \\ underwent assessments for best corrected \\ visual acuity, contrast sensitivity, \\ microperimetry, chromatic sensitivity, \\ macular thickness, and morphology using \\ spectral domain optical coherence \\ tomography (SD-OCT) and fluorescein \\ angiography at baseline. They were treated \\ with intravitreal Ozurdex at baseline and \\ monitored monthly with visual acuity and \\ SD-OCT assessments up to 36 weeks.
}

Laser and Retinal Research Unit, King's College Hospital NHS Foundation Trust, London, UK

Correspondence:

S Sivaprasad, Laser and Retinal Research Unit, King's College Hospital NHS Foundation Trust, Denmark Hill, Normandy Building, London SE5 9RS, UK. Tel: +44 (0)20 3299 4548; Fax: +44 (0)2032993738. E-mail: senswathi@aol.com

Received: 10 August 2013 Accepted in revised form: 22 November 2013 Published online: 3 January 2014
R Mathew, E Pearce, R Muniraju, A Abdul-Hey and S Sivaprasad
8 weeks followed by a sustained modest effect up to week 32 . The optimal re-treatment time point is at 20 weeks. Eye (2014) 28, 318-326; doi:10.1038/eye.2013.287; published online 3 January 2014

Keywords: macular oedema; visual outcomes; Ozurdex; optical coherence tomography; macular thickness

\section{Introduction}

Macular oedema (MO) is a common visionthreatening sequel of a variety of ocular conditions including diabetic retinopathy, central and branch retinal vein occlusion, and following cataract extraction. Hypo-reflective spaces representing focal coalescence of exudative fluid may appear localized to the inner or outer retina but frequently span the entire retinal thickness on optical coherence tomography (OCT). Chronic or severe MO may lead to temporary or permanent visual loss depending on the degree of strain or disruption of the microscopic intraretinal neural connections (transmission defects) and the intracellular damage suffered by the visual elements (transduction defects). ${ }^{1}$

Steroids inhibit the formation of both prostaglandins and leukotrienes and decrease intracellular and extracellular oedema by suppression of macrophage activity, vasoconstrictive effect, reduction of lymphokine production, ${ }^{2}$ and downregulation of production of vascular endothelial growth factor (VEGF). ${ }^{3}$ Several randomized clinical trials have reported good short-term efficacy data on intravitreal 
triamcinolone acetonide both in terms of improving visual acuity and reducing central retinal thickness (CRT) in patients with $\mathrm{MO}$ because of various retinal vascular diseases. ${ }^{4-6}$ However, the effect is short-lived and risks outweigh benefits. Intravitreal injections of triamcinolone are associated with elevated intraocular pressure (IOP) in up to $50 \%$ of injected eyes, cataract formation in $40 \%$ as well as sterile endophthalmitis.7,8

A number of corticosteroid-based intravitreal implants have been developed to provide a sustained release of drug obviating the need of repeated intravitreal injections. The Ozurdex (Allergan Inc., Irvine, CA, USA) drug delivery system is a sustained-release formulation for posterior-segment delivery of $700 \mu \mathrm{g}$ dexamethasone, made of a polylacticglycolic acid matrix. It received its market authorization in the European Union in 2010 for MO secondary to retinal vein occlusions. The recommended dose is one Ozurdex implant to be administered intravitreally to the affected eye and repeat doses to be considered when a patient experiences a response to treatment followed subsequently by a loss in visual acuity and in the physician's opinion may benefit from retreatment without being exposed to significant risk. The GENEVA trial was a 12-month study on Ozurdex for MO secondary to retinal vascular occlusions.9,10 The visual outcome of the study suggested that the effect of the drug waned after 12 weeks. The effect of Ozurdex on the macular morphology was not monitored every month in the study so it is difficult to draw conclusions on the anatomical impact of the drug. As visual acuity does not always correlate with clinical severity of $\mathrm{MO},{ }^{11}$ other visual functions such as contrast sensitivity, colour threshold, and retinal sensitivity may provide a better understanding of the effect of Ozurdex on MO.

The primary objective of this study was to evaluate the monthly changes in macular thickness with OCT in eyes treated with intravitreal Ozurdex for MO secondary to retinal vascular diseases, over 36 weeks, to evaluate the best re-treatment plan for Ozurdex. The secondary objectives were to assess the change from baseline in various visual functions and OCT parameters at 24 weeks.

\section{Setting}

The study was conducted in the Laser and Retinal Research Unit of King's College Hospital, London.

\section{Materials and methods}

This interventional, prospective, exploratory study (ISRCTN - 66216819) was conducted following approval from the Institutional Review Board Ethics Committee (11/H0718/6; NRES committee London Central) and all participants gave written informed consent. The study adhered to the principles of the Declaration of Helsinki.

\section{Patients}

Patients aged 18 years or above with a clinical diagnosis of MO secondary to diabetes, branch and central retinal vein occlusion, or pseudophakic or post-inflammatory cystoid MO and confirmed on Spectralis spectral domain-OCT (SD-OCT) and best corrected visual acuity (BCVA) in the study eye between 37 and 68 ETDRS letters were deemed eligible for the study. Patients with any other eye disease which could mask or contribute to $\mathrm{MO}$, or any ocular condition in the study eye that in the opinion of the investigator would prevent a 15-letter improvement in visual acuity (eg, severe macular ischemia, extensive macular laser scarring, or atrophy) were excluded. Patients with advanced glaucoma not adequately controlled by medicinal products alone, history of IOP elevation in response to steroid treatment in either eye that resulted in $\geq 10 \mathrm{~mm} \mathrm{Hg}$ increase in IOP from baseline with an absolute IOP $\geq 25 \mathrm{~mm} \mathrm{Hg}$, or required therapy with three or more anti-glaucoma medications were also excluded from the study. Systemic conditions that precluded trial entry included known uncontrolled systemic disease or current immunosuppressive disease, initiation of medical therapy for diabetes, or a change from oral hypoglycaemic agents to insulin therapy within 4 months before the screening visit and renal failure requiring haemodialysis or peritoneal dialysis within 6 months before screening visit. Women in the child bearing age underwent a pregnancy test and were advised to avoid conception during the 36 weeks of the study.

Patients were recruited into the trial from the retinal clinics in King's College Hospital NHS Foundation Trust from June 2011 to March 2012 and the last patient exited from the trial in December 2012.

\section{Assessments}

After written informed consent, patients underwent baseline examination of BCVA, dilated ophthalmic examination, tonometry, and Spectralis SD-OCT. The OCT volume scan was performed at every monthly visit on a $20 \times 20$ degree cube, with 49 raster lines, each containing 1064 pixels, separated by $125 \mu \mathrm{m}$. Macular stereo and four field fundus colour photographs and fundus fluorescein angiography were performed at baseline and week 24 only but could be repeated at the investigator's discretion.

All visual function tests were performed at baseline and repeated at week 24 . These included BCVA, contrast 
sensitivity, chromatic sensitivity, reading vision, and microperimetry. All patients were refracted by a certified examiner and BCVA for each eye was measured using standard ETDRS protocol at $4 \mathrm{~m}$ distance with a modified ETDRS distance chart. Visual acuity was scored as the total number of ETDRS letters read correctly.

Contrast sensitivity measurement was performed after visual acuity measurements, with the Pelli-Robson chart (Clement Clarke Inc., Harlow, UK) at a distance of $1 \mathrm{~m}$ and chart luminance of $80-120 \mathrm{~cd} / \mathrm{m}^{2}$.

After refraction, reading performance was measured by the certified examiner using a standardized protocol with the MNREAD acuity charts (Optelec, Vista, CA, USA). The reading acuity, maximum reading speed, and critical print size were calculated based on pre-defined formulae. ${ }^{12}$

Chromatic sensitivity was performed monocularly using the colour assessment and diagnosis test. This is a sensitive test that yields small colour detection thresholds. The test employs direction-specific, moving, chromatic stimuli embedded in a background of random, dynamic, luminance contrast noise. A four-alternative, forced-choice procedure is employed to measure the subject's thresholds for detection of colour signals in 16 directions in colour space, while ensuring that the subject cannot make use of any residual luminance contrast signals. $^{13}$

The Nidek Microperimeter (Nidek Technologies, Padova, Italy) was used to quantify macular sensitivity and fixation. It incorporates an eye tracker to compensate for eye movements and allows automated follow-up examination at the same retinal loci and also allows colour fundus image registration by infra-red camera ( $45^{\circ}$ field of view). The pupils were dilated before this test and the subject was dark adapted for $15 \mathrm{~min}$ before the test. The test was conducted according to pre-defined protocol: room darkened; a briefing trial test carried out initially; 5 min gap given between tests on each eye; all patients to have a $30 \mathrm{~s}$ fixation test; a $2^{\circ}$ red cross was used as fixation target, the test stimulus colour is white, Goldmann III size ( $26 \mathrm{~min}$ arc or $0.4^{\circ}$ ), and duration was $200 \mathrm{~ms}$. The background illumination was set at $1.27 \mathrm{~cd} / \mathrm{m}^{2}$. The intensity of the stimulus ranges from 0 to $20 \mathrm{db}$ where ' 0 ' represents the brightest luminance $\left(127 \mathrm{~cd} / \mathrm{m}^{2}\right)$. The perimetry strategy of the MP-1 starts at an initially defined threshold level $(12 \mathrm{~dB})$ for each stimulus. A 18 loci grid covering central $20^{\circ}$ was manually centered on fovea. We used the 4-2 threshold strategy. Fixation was measured for a period of $30 \mathrm{~s}$ once patients had located the cross.

The pattern of fixation was classified based on location and stability using the MP-1 software as recommended by Fujii et al. ${ }^{14}$

\section{Treatment}

All patients were treated at the baseline visit with intravitreal implant of $700 \mu \mathrm{g}$ dexamethasone, Ozurdex (Allergan Inc.) under aseptic precautions as per the protocol by a qualified investigator in a designated treatment room.

Re-treatment was allowed at weeks 16, 20, and 24 if the following criteria were met after an initial improvement (reduction) of macular thickness of at least $50 \mu \mathrm{m}$, average macular thickness increased by $100 \mu \mathrm{m}$ or more from the last visit and at least a five-letter drop in BCVA score from the previous visit, provided the patient had not experienced raised IOP above $30 \mathrm{~mm} \mathrm{Hg}$ following their first injection. Each patient was allowed to have a maximum of two treatments in this study.

\section{Outcome measures}

The primary outcome measure was the mean change in central subfield thickness (CST) at 4 weekly time points. Secondary OCT outcome measures included mean change in CRT and macular volume. Responders at each time point were defined in three ways: proportion of patients with CST $<300 \mu \mathrm{m}$, those with a decrease of CST by at least $100 \mu \mathrm{m}$, or $15 \%$ from baseline. Recurrence of oedema was defined as proportion of patients with an increase in CST by $100 \mu \mathrm{m}$ or $15 \%$ from lowest recorded CST. Rebound oedema was defined as an increase of at least $100 \mu \mathrm{m}$ from baseline after exposure to Ozurdex therapy. Non-responders were defined as those who persistently had CST at $\geq 300 \mu \mathrm{m}$ throughout the study.

Secondary visual function outcome measures included mean change in BCVA (ETDRS letters), proportion of patients with gain of $0,5,10$, and 15 ETDRS letters, or more (improvement) and proportion with loss of $<15$ ETDRS letters (stabilization), at weeks 24 and 36. The change in contrast sensitivity, colour vision thresholds, reading vision, microperimetry thresholds at $4^{\circ}$ and $12^{\circ}$, and change in fixation on microperimetry at 24 weeks were also recorded.

All serious adverse events (SAEs) and adverse events were recorded between baseline and week 36 .

\section{Analysis}

This is an exploratory study on changes in macular thickness after Ozurdex implant. So with an expected drop out rate of $5 \%$, we were expected to recruit 32 patients to have 30 patients with complete follow-up to fulfill our objectives. Efficacy assessment was performed on the full analysis set (the intent-to-treat population), which included all patients who received at least one application of study treatment and had at least one 
post-baseline assessment for BCVA. Missing data were imputed using the last-observation-carried-forward approach. Treatment-emergent adverse events were reported for all patients who received at least one application of study treatment and had at least one postbaseline safety assessment. Descriptive statistics for absolute values and changes from baseline were reported for each end point. For the variables, mean and standard deviations were used for continuous variables and counts and percentages for categorical variables. Analysis of variance was used for continuous, normally distributed variables. The categorical variables were examined using Fisher exact test or Pearson's $\chi^{2}$. Data were assumed significant if $P<0.05$ (SPSS V.17.0, SPSS, Chicago, IL, USA).

\section{Results}

\section{Demographics and baseline characteristics}

A total of 32 patients were evaluated and 30 patients were enrolled in the study. The baseline characteristics are shown in Table 1. All patients completed the followup and were included in the analysis. However, there were seven study appointments that were outside the window of the study visit. One patient did not have the second treatment despite being eligible for it because he was due for a surgery on the same day and preferred not to have his eye injection.

\section{Change in OCT parameters}

The mean decrease in CST and CRT from baseline was significant at all 4 weekly time points up to week 32 with steepest reduction observed at week 8 (Table 2). The lowest mean CST was observed at 8 weeks. The CST remained $100 \mu \mathrm{m}$ less than baseline until week 16 . Although the macular volume decreased from baseline at all time points, the change was minimal from week 16 . The lowest mean macular volume was achieved at week 12 . The changes in these parameters were no different when the DMO sub-group was analysed as a sub-group.

\section{Change in visual acuity}

The mean change in visual acuity from baseline was significant until week 12 (Table 2). The maximal mean visual acuity improvement was observed at 12 weeks. The number of patients with a gain of $0,5,10$, or 15 letters or more at 24 weeks were 20 (66\%), 17 (56.6\%), $12(40 \%)$, and $6(20 \%)$, respectively, and the corresponding proportions at 36 weeks were 17 (56.6\%), $14(46.6 \%), 11(36.6 \%)$, and $3(10 \%)$. The number of patients who lost less than 15 letters were $26(86.6 \%)$ and
Table 1 Patient demographics and baseline characteristics

\begin{tabular}{lc}
\hline Mean age (SD), years & $62 \pm 3.54$ \\
Range, years & $41-91$ \\
Male, $n(\%)$ & $16(53.3)$ \\
Race, $n$ (\%) & \\
$\quad$ Caucasian & $20(66.7)$ \\
$\quad$ Afrocaribbean & $8(26.7)$ \\
Asian & $2(6.7)$ \\
& \\
Causes of macular edema, $n(\%)$ & \\
$\quad$ Diabetic macular edema & \\
$\quad$ Mild and moderate NPDR & $10(33.3)$ \\
$\quad$ Severe NPDR & $7(23.3)$ \\
$\quad$ Treated proliferative diabetic retinopathy & $7(23.3)$ \\
Central retinal vein occlusion & 0 \\
Branch retinal vein occlusion & $5(16.7)$ \\
Inflammatory & 0 \\
Pseudophakic & $1(3.3)$ \\
Smokers, $n$ (\%) & $11(36.7)$ \\
& \\
Mean HbA1C (SD) in diabetics & \\
$\quad \leq 8 \%, n$ (\%) & $15(50)$ \\
>8\%, $n$ (\%) & $9(30)$ \\
Hypertensives on treatment, $n(\%)$ & $12(40)$ \\
Previous glaucoma medications & 0 \\
Pseudophakia, $n$ (\%) & $8(26.7)$ \\
Previous macular laser, $n(\%)$ & $18(60)$ \\
Mean number of laser treatments, $n$ (SD) & $2.3 \pm 1.6$ \\
Mean ETDRS letter score (SD) & $56.1 \pm 9.65$ \\
$<54$ letters, $n$ (\%) & $12(40)$ \\
$\geq 54$ letters, $n$ (\%) & $18(60)$ \\
\hline
\end{tabular}

Abbreviations: ETDRS, early treatment diabetic retinopathy study; HbA1C, glycosylated haemoglobin; NPDR, non-proliferative diabetic retinopathy.

$28(93.3 \%)$ at 24 and 36 weeks, respectively. One patient lost 26 letters at 20 weeks due to anterior uveitis and then recovered to baseline visual acuity by 36 weeks. Another patient developed vitreous haemorrhage because of posterior vitreous detachment at 32 weeks, but improved to 41 letters by 36 weeks. In the DMO sub-group, the mean change in visual acuity from baseline was significant until week 20 . The maximal visual acuity gain was achieved at 12 weeks as in the whole cohort, but the mean visual acuity gain was 1.2 letters more (10.4 vs 9.2) and was sustained up to 20 weeks (data not shown).

\section{Change in IOP}

The IOP increased from mean value of $15.6+2.8 \mathrm{~mm} \mathrm{Hg}$ at baseline to $17.1+2.8 \mathrm{~mm} \mathrm{Hg}$ at 4 weeks,

$18.1+1 \mathrm{~mm} \mathrm{Hg}$ at 8 weeks, $18.1+2 \mathrm{~mm} \mathrm{Hg}$ at 12 weeks, $16.7+3 \mathrm{~mm} \mathrm{Hg}$ at 16 weeks, and $16.1+2 \mathrm{~mm} \mathrm{Hg}$ by 20 weeks. The IOP showed a rise to $17+2 \mathrm{~mm} \mathrm{Hg}$ by 24 and 28 weeks and falling to $16+2 \mathrm{~mm} \mathrm{Hg}$ by 36 weeks 
Table 2 Changes in OCT parameters and visual acuity per month

\begin{tabular}{|c|c|c|c|c|c|c|c|c|c|c|}
\hline & Mean CST & $\begin{array}{l}\text { CST change } \\
\text { from baseline }\end{array}$ & $\mathrm{P}$ value & $\begin{array}{c}\text { CST change } \\
\text { from previous } \\
\text { visit }\end{array}$ & $\mathrm{P}$ value & $\begin{array}{c}\text { Mean visual } \\
\text { acuity }\end{array}$ & $\begin{array}{l}\text { Change in } \\
\text { visual acuity } \\
\text { from baseline }\end{array}$ & P value & $\begin{array}{c}\text { Change in visual } \\
\text { acuity from } \\
\text { previous visit }\end{array}$ & $\mathrm{P}$ value \\
\hline Baseline & $527.4+47.5$ & & & & & $56.10+9.7$ & & & & \\
\hline 4 Weeks & $337.1+28.6$ & $-190.3+54.6$ & 0.000 & -190.33 & 0.000 & $64.28+11.6$ & $8.18 \pm 5.5$ & 0.000 & $8.18+5.5$ & 0.000 \\
\hline 8 weeks & $301+27$ & $-226.4+53.4$ & 0.000 & -36.07 & 0.001 & $64.93+12.7$ & $8.83 \pm 5.8$ & 0.004 & $0.65+2.2$ & 0.804 \\
\hline 12 Weeks & $347.1+49.4$ & $-180+68$ & 0.000 & 46.07 & 0.020 & $65.34+14.2$ & $9.2 \pm 6.2$ & 0.005 & $0.41+3.1$ & 0.841 \\
\hline 16 Weeks & $416.9+61.7$ & $-110+68.5$ & 0.002 & 69.83 & 0.000 & $61.55+14.5$ & $5.45 \pm 6.4$ & 0.096 & $-3.79+2.4$ & 0.005 \\
\hline 20 Weeks & $408.1+60$ & $-94.3+75.7$ & 0.003 & 19.31 & 0.184 & $60.21+15.3$ & $4.1 \pm 6.6$ & 0.224 & $-1.34+3.7$ & 0.132 \\
\hline 24 Weeks & $406.1+58.2$ & $-121+73.8$ & 0.002 & -16.54 & 0.600 & $61.67+14.9$ & $5.5 \pm 6.5$ & 0.092 & $1.46+2.6$ & 0.458 \\
\hline 28 Weeks & $419.2+53$ & $-108.1+69.5$ & 0.003 & -0.43 & 0.983 & $64.13+13.9$ & $8+6.1$ & 0.012 & $2.46+2.7$ & 0.016 \\
\hline 32 Weeks & $437.4+53$ & $-89.6+69.9$ & 0.013 & 18.48 & 0.224 & $61.82+12.9$ & $3.58 \pm 7.2$ & 0.328 & $-2.31+1.2$ & 0.008 \\
\hline 36 Weeks & $457.3+57$ & $-70.1+73$ & 0.059 & 19.58 & 0.091 & $60.33+13.2$ & $4.2 \pm 6$ & 0.163 & $-1.49+2.5$ & 0.790 \\
\hline
\end{tabular}

$P<0.05$ is given in bold.

Abbreviation: CST, central sub-field thickness.

\section{Responder profile}

Table 3 shows the proportion of patients that may be defined as responders based on the three definitions of responders. The maximum number of responders was observed at 8 weeks irrespective of the definitions used to define responders. Proportion of patients with a CST of less than $300 \mu \mathrm{m}$ reduced significantly by 16 weeks. However, if a reduction of $15 \%$ or $100 \mu \mathrm{m}$ is used to define response, approximately $45 \%$ qualify as responders until week 28 . Rebound oedema from 20 weeks was observed in less than $10 \%$ of the sample. Recurrence of oedema based on an increase in CST by $100 \mu \mathrm{m}$ or by $15 \%$ from lowest recorded CST was noted as early as 12 weeks and continued to increase until 36 weeks. The number of patients with a decrease of five letters in BCVA gradually increased to $46.7 \%$ by 20 weeks.

\section{Other visual function changes at 24 weeks}

All visual functions except contrast sensitivity improved significantly at 24 weeks compared with baseline (Table 4).

\section{Re-treatment criteria}

Proportion of patients that required a second injection based on our re-treatment criteria was $16.6 \%$ (5/30). They were all patients with DMO. Two patients each were treated at 16 weeks and 24 weeks and one at 20 weeks. The changes in visual acuity and CST for the whole cohort were compared with those who received only a single treatment at baseline. The patterns of changes in visual acuity and CST over time were similar as shown in Figure 1. However, we can infer that patients who required second treatment had poorer potential for visual gain.

\section{Safety events}

Two patients $(6.7 \%)$ required IOP-lowering medications and one patient developed vitreous haemorrhage secondary to posterior vitreous detachment. A fifth of the study cohort suffered serious SAEs but none were related to the study medication. Six patients had SAEs, none of them were related to the Ozurdex injection. These included fracture wrist, fracture leg, surgery for carpal tunnel syndrome, scheduled surgery for aortic valve replacement, hyperkalaemia in one patient, and injection (insulin) site infection in another patient (Table 5). One patient lost 26 letters at 20 weeks because of anterior uveitis and then recovered to baseline visual acuity by 36 weeks.

\section{Discussion}

This study shows that the maximal efficacy in terms of lowest recorded mean CST after a single dose of Ozurdex was at 8 weeks. The most dramatic reduction in mean CST of approximately $200 \mu \mathrm{m}$ was noted at 4 weeks. The optimal re-treatment time point was at 20 weeks.

The pharmacokinetic effects of Ozurdex have been studied in normal monkey eyes. The pharmacokinetics of Ozurdex in the vitreous and retina of monkey eyes demonstrated two distinct phases that corresponded to the observed fragmentation of the implant. A high initial drug concentration was observed for 60 days followed by a period of low concentration up to 210 days. The drug was not detected from days 240 to $270 .{ }^{15}$ The use of central macular thickness to model changes of intraocular drug concentrations has been previously described. Aubren et al demonstrated a population 
Table 3 Responder profile

\begin{tabular}{|c|c|c|c|c|c|c|c|c|}
\hline \multicolumn{4}{|c|}{ Responders (\%) } & \multicolumn{2}{|c|}{ Recurrence (\%) } & \multirow[b]{2}{*}{$F$} & \multirow[b]{2}{*}{$G$} & \multirow[b]{2}{*}{$H$} \\
\hline Time & $A$ & $B$ & C & $D$ & E & & & \\
\hline Week 4 & $12 / 30(40)$ & $20 / 30(66.7)$ & $18 / 30(60)$ & 0 & 0 & $4 / 30(13.3)$ & 4/30 (13.3) & 0 \\
\hline Week 8 & $19 / 30(63.3)$ & 24/30 (80) & $21 / 30(70)$ & 0 & 0 & 5/30 (16.6) & 6/30 (20) & 0 \\
\hline Week 12 & $14 / 30(46.6)$ & $19 / 30(63.3)$ & $17 / 30(56.7)$ & $5 / 30(16.7)$ & 4/30 (13.3) & $4 / 30(13.3)$ & $8 / 30(26.7)$ & 0 \\
\hline Week 16 & $5 / 30(16.6)$ & $14 / 30(46.7)$ & $14 / 30(46.7)$ & $12 / 30(40)$ & 9/30 (30) & $7 / 30(23.3)$ & $15 / 30(50)$ & $8 / 30(26.7)$ \\
\hline Week 20 & $3 / 30(10)$ & $14 / 30(46.7)$ & $11 / 30(36.7)$ & $16 / 30(53.3)$ & $12 / 30(40)$ & $9 / 30(30)$ & $20 / 30(66.7)$ & $13 / 30(43.3)$ \\
\hline Week 24 & $6 / 30(20)$ & $15 / 30(50)$ & $12 / 30(40)$ & $16 / 30(53.3)$ & $13 / 30(43.3)$ & $7 / 30(23.3)$ & $18 / 30(60)$ & $11 / 30(36.7)$ \\
\hline Week 28 & $7 / 30(23.3)$ & $14 / 30(46.7)$ & $14 / 30(46.7)$ & $14 / 30(46.7)$ & $9 / 30(30)$ & $7 / 30(23.3)$ & $14 / 30(46.7)$ & $8 / 30(26.7)$ \\
\hline Week 32 & $4 / 30(13.3)$ & $11 / 30(36.7)$ & $10 / 30(33.3)$ & $15 / 30(50$ & $12 / 30(40)$ & $10 / 30(33.3)$ & $17 / 30(56.7)$ & $9 / 30(30)$ \\
\hline Week 36 & $6 / 30(20)$ & $11 / 30(36.7)$ & $8 / 30(26.7)$ & $19 / 30(63.3)$ & $15 / 30(50)$ & $11 / 30(36.7)$ & $22 / 30(73.3)$ & $16 / 30(53.3)$ \\
\hline
\end{tabular}

(A) Proportion of patients with CST (central sub-field thickness) $<300 \mu \mathrm{m}$ (responder).

(B) Proportion of patients with decrease of CST by $100 \mu \mathrm{m}$ from baseline (responder).

(C) Proportion of patients that showed a decrease of CST by at least 15\% from baseline (responders).

(D) Proportion of patients with increase CST by $100 \mu \mathrm{m}$ from lowest recorded CST (recurrence)

(E) Proportion of patients with increase CST by 15\% from lowest recorded CST (recurrence).

(F) Proportion of patients with drop of BCVA (best corrected visual acuity) by 5 letters or more from baseline.

(G) Proportion of patients with drop of BCVA by 5 letters or more from best recorded BCVA.

(H) Proportion of patients with a drop of BCVA by 5 letters or more from best recorded BCVA and an increase CST by $100 \mu \mathrm{m}$ from lowest recorded CST.

Table 4 Secondary visual function outcomes at 24 weeks

\begin{tabular}{|c|c|c|c|c|}
\hline & Baseline & 24 Weeks & Mean change/SD & $\mathrm{P}$ value \\
\hline Contrast sensitivity & $29.8+3.7$ & $30.7+5.3$ & $0.9+1.6$ & 0.149 \\
\hline Reading acuity & $0.51+0.19$ & $0.41+0.28$ & $0.1+0.12$ & 0.034 \\
\hline MRS & $164.3+53.8$ & $185.9+71.9$ & $21.6+18.1$ & 0.039 \\
\hline CPS & $0.988+0.19$ & $0.873+0.26$ & $0.115+0.15$ & 0.035 \\
\hline Ret sensitivity $4^{\circ}$ & $4.8+4.5$ & $9.2+5.8$ & $4.4+4.1$ & $<0.0001$ \\
\hline Ret sensitivity $12^{\circ}$ & $6.1+4.5$ & $10.2+5.5$ & $4.1+4.3$ & $<0.0001$ \\
\hline Mean tritan threshold (SD) & $20.6+11.7$ & $16.4+11.6$ & $4.2+10.1$ & 0.012 \\
\hline Mean protan threshold (SD) & $14.7+5.1$ & $12.9+6.6$ & $1.8+5$ & 0.073 \\
\hline Fixation location, $n$ & 28 & 28 & & \\
\hline PC, $n(\%)$ & $13(46.4)$ & $12(40)$ & & $>0.05$ \\
\hline POC, $n(\%)$ & $3(10.7)$ & $6(20)$ & & \\
\hline PE, $n(\%)$ & $12(40)$ & $10(33)$ & & \\
\hline Fixation stability, $n$ & 28 & 28 & & \\
\hline Stable, $n(\%)$ & $19(67.9)$ & $16(53.3)$ & & \\
\hline RUS, $n(\%)$ & $7(25)$ & $11(36.6)$ & & $>0.05$ \\
\hline Unstable, $n(\%)$ & $2(7.1)$ & $1(3.3)$ & & \\
\hline
\end{tabular}

Abbreviations: CPS, critical print size; MRS, mean reading speed; PC, predominantly central; PE, predominantly eccentric; POC, poor central; RUS, relatively unstable.

$P<0.05$ is shown in bold.

pharmacokinetic-pharmacodynamic modelling to understand the elimination rate of triamcinolone based on the changes in central macular thickness. ${ }^{16}$

In comparison, in this study, if the reduction of CST from baseline is considered a surrogate marker for the overall durability of the drug concentration in the vitreous and retina from baseline, the findings of this study mirror the effects in normal monkey eyes in that the initial high drug concentration phase lasts 8 weeks. The CST then remains below baseline until about week 36 , suggesting that a second phase of lower concentration of Ozurdex continues to provide a sustained drying effect of the retina.
If we consider the rate of reduction of CST between visits as the marker of remaining drug concentration at each visit, the maximal effect of the drug is at 4 weeks with a dramatic mean reduction of $190 \mu \mathrm{m}$ and a further $36 \mu \mathrm{m}$ reduction at 8 weeks supporting the period of initial high drug concentration. The lowest recordable mean CST was also at 8 weeks.

Therefore, this study substantiates the pharmacokinetic and pharmcacodynamic studies of Ozurdex in normal monkeys by using three surrogate markers of drug concentration: change in CST from baseline, change in CST from previous visit, and lowest 


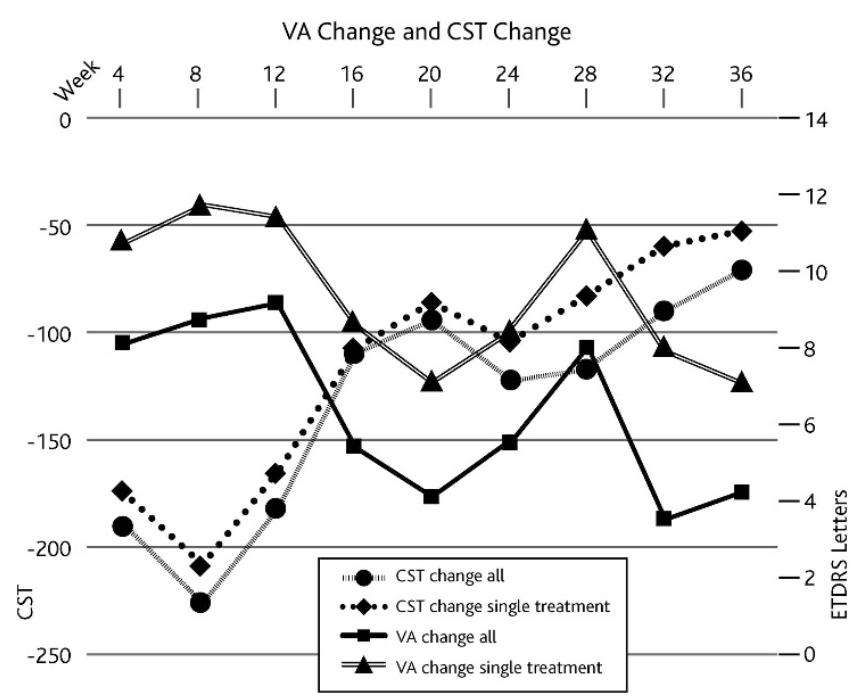

Figure 1 Graph showing the change in central sub-field thickness (CST) and visual acuity (VA) over time in the whole study cohort and those that received a single Ozurdex injection only.

Table 5 Adverse events

\begin{tabular}{lc}
\hline Any AE related to treatment, $n(\%)$ & $2(6.7)$ \\
Any SAE, $n(\%)$ & $6(20)$ \\
Any SAE related to treatment, $n(\%)$ & 0 \\
& \\
Proportion requiring IOP lowering medication, $n(\%)$ & $2(6.7)$ \\
1 IOP-lowering medication & 0 \\
2 IOP-lowering medications & 0 \\
Proportion requiring cataract surgery & \\
Other selected adverse events, $n(\%)$ & 0 \\
Endophthalmitis & 1 \\
Anterior uveitis & $1(3.3 \%)$ \\
Vitreous haemorrhage & 0 \\
Retinal detachment & \\
Systemic adverse events & \\
Deaths & \\
Non-fatal MI/CVA & 0 \\
\end{tabular}

Abbreviations: AE, adverse event; IOP, intraocular pressure; $\mathrm{SAE}$, serious adverse event.

recordable CST. These observations suggest that unlike other biodegradeable implants with a triphasic response curve, Ozurdex has a biphasic response of an early burst because of high concentration of the drug that is followed by a plateau that last about 32 weeks.

The anatomical changes observed in this study highlight the strengths and weaknesses of Ozurdex in the treatment of $\mathrm{MO}$ because of retinal vascular diseases. The immediate drying effect of the macula was noted in all but one patient indicating that it may be useful not only as first-line drying agent but also as sequential or combination therapy with anti-VEGF therapy. However, the recurrence of oedema by 16-20 weeks is a disadvantage as early re-treatment may also cause increase rates of elevated IOP and cataract as reported with intravitreal triamcinolone. Given that the proportion of patients with a reduction of five letters or more in BCVA associated with an increase in CST of $100 \mu \mathrm{m}$ or more from lowest recorded value was highest at 20 weeks, it seems optimal to state that a 5 monthly re-treatment is appropriate if the re-treatment criteria are met.

Another point observed in this study is the ideal choice of re-treatment criteria. Only five patients met the re-treatment criteria of more than five-letter loss with an increase in $100 \mu \mathrm{m}$ from the previous visit. However, if an increase of $100 \mu \mathrm{m}$ from lowest recorded CST was used as the re-treatment criteria approximately $50 \%$ of the participants would have been re-treated at 16-20 weeks. Given that $100 \mu \mathrm{m}$ is an arbitrary figure, an individualized re-treatment criterion may be defined as an increase by $15 \%$ from lowest recorded CST. This re-treatment would mean that $40 \%$ of the participants would have required treatment at 20 weeks. However, if a five-letter drop in visual acuity and an increase in $100 \mu \mathrm{m}$ from lowest recorded CST are taken into account, the optimal re-treatment time point is at 20 weeks. Earlier re-treatment based on changes in CST alone may be associated with higher prevalence of cataract and raised IOPs.

Rebound oedema after Ozurdex has been reported previously. ${ }^{17}$ This effect was minimal in this study with a maximum of three patients showing increased CST above baseline after an initial reduction. There was one patient with an increase in CST more than baseline with no evidence of any reduction in CST following treatment. This observation shows that the drying effect of Ozurdex is almost universal for most retinal vascular diseases despite the cause of the MO. 
The maximum gain of 9.24 letters is observed at 12 weeks. This pattern of visual gain with Ozurdex closely resembles the effect of loading phase of 3 monthly antiVEGF injections. Therefore, Ozurdex may be used as firstline treatment as a drying agent instead of three antiVEGF injections. It is promising to observe that all visual functions tested in this study except contrast sensitivity also showed significant improvement at 24 weeks as this indicates a positive effect on the retinal neuronal function probably due to a realignment of neuronal structures induced by the drying effect of Ozurdex. A direct neurtrophic effect of Ozurdex cannot be ruled out.

In conclusion, this study shows that Ozurdex is an effective therapy to improve visual function and macular thickness in patients with $\mathrm{MO}$ because of retinal vascular disease up to 36 weeks. If re-treatment is indicated, the time point of 20 weeks seems to represent a reasonable compromise in terms of effectiveness and side-effects of Ozurdex.

\footnotetext{
What was known before

- The re-treatment time point for Ozurdex in previous clinical trials for macular oedema was at 6 months.

- The maximal visual gain was reported at 2 months.

- The complications of cataract and glaucoma in Ozurdex were low compared with intravitreal triamcinolone.
}

\section{What this study adds}

- The maximal effect on reduction of central sub-field thickness is at 8 weeks.

- The maximal effect on visual acuity is at 12 weeks.

- The optimal re-treatment time point is at 20 weeks.

\section{Conflict of interest}

$\mathrm{R}$ Mathew has received travel grants and speaker fees from Allergan. S Sivaprasad has received research grants, travel grants, and participated in advisory boards of Novartis, Pfizer, Allergan, Alimera services, and Bayer. The remaining authors declared no conflict of interest.

\section{Acknowledgements}

We thank Rashmi Akshikar, Roopa Vemala, Matthew Richardson, Richard Leung for assistance in conducting the trial. We thank Allergan Ltd for funding this project and D J Simon for statistical support. Institutional Review Board Ethics Committee (11/H0718/6) (NRES committee London Central).

\section{References}

1 Pelosini L, Hull CC, Boyce JF, McHugh D, Stanford MR, Marshall J. Optical coherence tomography may be used to predict visual acuity in patients with macular edema. Invest Ophthalmol Vis Sci 2011; 52(5): 2741-2748.

2 Abe T, Hayasaka S, Nagaki Y, Kadoi C, Matsumoto M, Hayasaka Y. Pseudophakic cystoid macular edema treated with high-dose intravenous methylprednisolone. J Cataract Refract Surg 1999; 25(9): 1286-1288.

3 Binz N, Graham CE, Simpson K, Lai YK, Shen WY, Lai CM et al. Long-term effect of therapeutic laser photocoagulation on gene expression in the eye. Faseb J 2006; 20(2): 383-385.

4 Scott IU, Ip MS, VanVeldhuisen PC, Oden NL, Blodi BA, Fisher $\mathrm{M}$ et al. A randomized trial comparing the efficacy and safety of intravitreal triamcinolone with standard care to treat vision loss associated with macular Edema secondary to branch retinal vein occlusion: the Standard Care us Corticosteroid for Retinal Vein Occlusion (SCORE) study report 6. Arch Ophthalmol 2009; 127(9): 1115-1128.

5 Martidis A, Duker JS, Greenberg PB, Rogers AH, Puliafito CA, Reichel E et al. Intravitreal triamcinolone for refractory diabetic macular edema. Ophthalmology 2002; 109(5): 920-927.

6 Young S, Larkin G, Branley M, Lightman S. Safety and efficacy of intravitreal triamcinolone for cystoid macular oedema in uveitis. Clin Exp Ophthalmol 2001; 29(1): 2-6.

7 Jonas JB, Kreissig I, Sofker A, Degenring RF. Intravitreal injection of triamcinolone for diffuse diabetic macular edema. Arch Ophthalmol 2003; 121(1): 57-61.

8 Loewenstein A, Goldstein M. Intravitreal triamcinolone acetonide for diabetic macula edema. Isr Med Assoc J 2006; 8(6): 426-427.

9 Haller JA, Bandello F, Belfort Jr. R, Blumenkranz MS, Gillies M, Heier J et al. Randomized, sham-controlled trial of dexamethasone intravitreal implant in patients with macular edema due to retinal vein occlusion. Ophthalmology 2010; 117(6): 1134-1146.

10 Haller JA, Bandello F, Belfort Jr R, Blumenkranz MS, Gillies M, Heier J et al. Dexamethasone intravitreal implant in patients with macular edema related to branch or central retinal vein occlusion twelvemonth study results. Ophthalmology 2011; 118(12): 2453-2460.

11 Browning DJ, Glassman AR, Aiello LP, Beck RW, Brown DM, Fong DS et al. Relationship between optical coherence tomography-measured central retinal thickness and visual acuity in diabetic macular edema. Ophthalmology 2007; 114(3): $525-536$.

12 Legge GE, Ross JA, Luebker A, LaMay JM. Psychophysics of reading. VIII. The Minnesota Low-Vision Reading Test. Optom Vis Sci 1989; 66(12): 843-853.

13 Connolly DM, Barbur JL, Hosking SL, Moorhead IR. Mild hypoxia impairs chromatic sensitivity in the mesopic range. Invest Ophthalmol Vis Sci 2008; 49(2): 820-827.

14 Fujii GY, De Juan Jr E, Humayun MS, Sunness JS, Chang TS, Rossi JV. Characteristics of visual loss by scanning laser ophthalmoscope microperimetry in eyes with subfoveal choroidal neovascularization secondary to age-related macular degeneration. Am J Ophthalmol 2003; 136(6): 1067-1078.

15 Chang-Lin JE, Attar M, Acheampong AA, Robinson MR, Whitcup SM, Kuppermann BD et al. Pharmacokinetics and 
pharmacodynamics of a sustained-release dexamethasone intravitreal implant. Invest Ophthalmol Vis Sci 2011; 52(1): 80-86.

16 Audren F, Tod M, Massin P, Benosman R, Haouchine B, Erginay A et al. Pharmacokinetic-pharmacodynamic modeling of the effect of triamcinolone acetonide on central macular thickness in patients with diabetic macular edema. Invest Ophthalmol Vis Sci 2004; 45(10): 3435-3441.

17 Querques L, Querques G, Lattanzio R, Gigante SR, Del Turco C, Corradetti G et al. Repeated intravitreal dexamethasone implant (Ozurdex(R)) for retinal vein occlusion. Ophthalmologica 2013; 229(1): 21-25. 